\title{
Implementation of an Imaging Spectrometer for Localization and Identification of Radioactive Sources
}

\author{
H. Lemaire ${ }^{\mathrm{a}}$, R. Abou Khalil ${ }^{\mathrm{b}}$, K. Amgarou ${ }^{\mathrm{b}}$, J.-C. Angélique $^{\mathrm{c}}$, F. Bonnet $^{\mathrm{d}}$, \\ D. De Toro ${ }^{\text {b }}$, F. Carrela , O. Giarmana ${ }^{\mathrm{d}}$, M. Gmar ${ }^{\mathrm{a}}$, N. Menaa ${ }^{\mathrm{b}}$, Y. Menesguen ${ }^{\mathrm{a}}$,

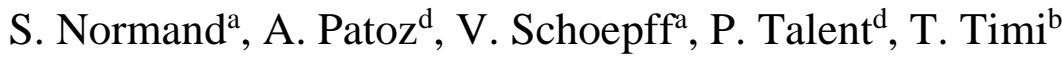 \\ ${ }^{\mathrm{a} C E A}$, LIST, Gif-sur-Yvette, F-91191, France \\ ${ }^{\mathrm{b}}$ CANBERRA, 1 rue des hérons, Saint-Quentin-en-Yvelines, F-78182, France \\ ${ }^{\mathrm{c}}$ LPC, 6 boulevard du Maréchal Juin, F-14050, France \\ ${ }^{\mathrm{d}}$ CANBERRA, 10 route de Vauzelles, Loches, F-37600, France
}

\section{Corresponding author: H. Lemaire, hermine.lemaire@cea.fr Telephone: 0033169083575}

Abstract

Spatial localization of radioactive sources is currently a main issue interesting nuclear industry as well as homeland security applications and can be achieved using gamma cameras. For several years, CEA LIST has been designing a new system, called GAMPIX, with improved sensitivity, portability and ease of use. The main remaining limitation of this system is the lack of spectrometric information, preventing the identification of radioactive materials. This article describes the development of an imaging spectrometer based on the GAMPIX technology. Experimental tests have been carried out according to both spectrometric methods enabled by the pixelated Timepix chip used in the GAMPIX gamma camera. The first method is based on the size of the impacts produced by a gamma-ray energy deposition in the detection matrix. The second one uses the Time over Threshold (ToT) mode of the Timepix chip and deals with time spent by pulses generated by charge preamplifiers over a user-specified threshold. Both energy resolution and sensitivity studies demonstrated the superiority of the ToT approach which will consequently be further explored. Energy calibration, tests of different pixel sizes for the Timepix chip and use of the Medipix 3 chip are future milestones to improve performances of the newly implemented imaging spectrometer.

Keywords

\section{Gamma imaging; GAMPIX; Timepix; imaging spectrometer; Time over Threshold}

\section{Introduction}

Spatial localization of radioactive sources is currently a main issue interesting nuclear industry (nuclear power plants security, decommissioning of nuclear facilities, radiationprotection) as well as homeland security applications (controls, post-accidental interventions) 
[1] [2]. Gamma imaging is a very interesting technique to achieve this spatial localization by enabling superimposition of visible and gamma pictures using dedicated devices called gamma cameras.

Spatial localization can be achieved using Compton scattering or coded masks. Compton approach includes two steps: the scattering of the incident photon and its full absorption. From the path of each incident photons one can determine cones from which it could have been emitted. The radioactive source is located at the intersection of all the rebuilt cones. Two sensors are usually involved in these systems but gamma cameras based on a single sensor also exist. We can give the example of the recently industrialized Polaris-H system [3] [4]. In this new gamma camera, the depth of interaction required to determine the path is obtained from the cathode-to-anode signal ratio (CAR) or from drift time information. Because photons have to deposit energy in two successive detectors, the Compton approach is mainly dedicated to photons above $200 \mathrm{keV} \mathrm{[3].} \mathrm{In} \mathrm{the} \mathrm{rest} \mathrm{of} \mathrm{the} \mathrm{article,} \mathrm{we} \mathrm{will} \mathrm{focus} \mathrm{on} \mathrm{gamma} \mathrm{cameras}$ using coded masks.

Current industrial gamma cameras based on coded masks can be considered as first generation because they are based on scintillator detectors. Much progress was made since the design of the first gamma camera by Hal Anger (Berkeley University, California) in the last's 50 for medical applications [5]: digitalization of data processing [6], replacement of the pinholes used for spatial localization (CARTOGAM, CEA LIST [7]) by multiple hole collimators (Fixed Multiple hole Collimated Camera, University of Michigan [8]) and MURA coded masks (RadCam, Radiation Monitoring Devices Inc. [9]), etc. In the 90's, continuous scintillators moved into pixelated scintillators (CSPD-2, University of Michigan [10]; RMDPinhole, Radiation Monitoring Devices Inc. [11]). At the same time, semiconductor detectors were developed [6]. Such detectors intended to improve both spatial and energy resolution by enabling direct conversion from gamma photons to electrical charge. First gamma cameras integrating semiconductor detectors present some limitations because of small detection surfaces resulting in small fields of view $([12,13])$ and obligation of cooling the detector when using materials such as germanium [12]. The progressive development of pixelated CdTe or CdZnTe substrates hybridized to ASICs [14, 15, 16, 17] opened the way to a second generation of gamma cameras operating at room temperature.

In this context, CEA LIST designed a second generation system, named GAMPIX [1, 18, 19]. GAMPIX's body integrates three main building blocks:

The detection system is a $1 \mathrm{~mm}$ thick CdTe substrate bump-bonded to a pixelated readout chip called Timepix [17] and developed by the CERN. In $1.4 \mathrm{~cm}^{2}$, the Timepix chip integrates 256 pixels by 256 pixels, $55 \mu \mathrm{m}$ side, with independent shaping and processing chains. collimator for spatial localization [20]. It is characterized by its number of holes (rank) and its thickness.

80 Finally, the USB module enables plug-and-play connection of the gamma camera with the 
GAMPIX is currently under industrialization by AREVA CANBERRA (the industrial system is named iPix, see Fig. 1). Compared to CARTOGAM, which is the current AREVA CANBERRA industrial system, GAMPIX presents three main improvements:

The first one is the low-energy (below $100 \mathrm{keV}$ ) sensitivity with a gain of five decades in comparison with CARTOGAM. GAMPIX is able to detect in $1 \mathrm{~s} \mathrm{a}{ }^{241} \mathrm{Am}$ radioactive source generating a dose rate of $0.25 \mu \mathrm{Sv} \mathrm{h}^{-1}$ in the vicinity of the gamma camera. For this reason, GAMPIX is a performing tool for plutonium detection during decommissioning operations $\left({ }^{241} \mathrm{Am}\right.$ being a feature of the presence of plutonium). GAMPIX efficiency decreases at high energy because of both the small detection volume $\left(0.1982 \mathrm{~cm}^{3}\right.$ of CdTe against $5 \mathrm{~cm}^{3}$ of CsI(Tl) for CARTOGAM) and the non-perfect filtering achieved by the coded mask. For this reason, $20 \mathrm{~s}$ are needed to detect a ${ }^{137} \mathrm{Cs}$ radioactive source with $2.5 \mu \mathrm{Sv} \cdot \mathrm{h}^{-1}$ dose rate and $60 \mathrm{~s}$ for a ${ }^{60} \mathrm{Co}$ source giving a dose rate of $3.8 \mu \mathrm{Sv}^{-1} \mathrm{~h}^{-1}$. However, it is important to emphasize that, by adapting the characteristics of the coded mask, GAMPIX is able to cover an energy range from ${ }^{241} \mathrm{Am}$ to ${ }^{60} \mathrm{Co}$ with better performances than CARTOGAM even at high energy (see [1] for results obtained in Nuclear Power Plants).

The second point is the portability facilitated by the reduction of the weight. CARTOGAM, which is the lightest system currently on the market, and GAMPIX respectively weight $15 \mathrm{~kg}$ and $2 \mathrm{~kg}$. The difference is mainly due to the shielding required by the scintillation detector of CARTOGAM.

Finally, the third point deals with the ease of use and deployment of GAMPIX in comparison with CARTOGAM. GAMPIX uses for instance only one cable for camera management, data transmission and power supply.

Besides, GAMPIX has a field of view of $50^{\circ}$. The angular resolution, which refers to the minimal angle between two radioactive sources to be separated in the decoded image, reaches down to $2^{\circ}$ for a ${ }^{241} \mathrm{Am}$ radioactive source.

GAMPIX applications benefit from its characteristics. Thanks to its great portability, it can easily be deployed in nuclear power plants in order to control, for instance, the correct position of lead shielding dedicated to the radiation protection of operators. Regarding nuclear facilities decommissioning, GAMPIX is able to provide an accurate localization of hot spots (for instance, in pipes) for targeted decommissioning enabling both reduction of operations duration and waste volume to be stored. The sensitivity of GAMPIX and its easy deployment by non-expert end-users enable its use for fast control of luggage (airports) and containers (ports) for homeland security applications. Finally, for post-accidental interventions, GAMPIX can help the first responders to quickly identify dangerous areas in Fukushima type environments. Experimental results illustrating these applications can be found in the Reference [1].

In its current version, the main limitation of the GAMPIX gamma camera is the lack of spectrometric information, preventing the identification of radioactive material. Thus, dose rate calculation needs an assumption on the nature of radionuclides and it is impossible to identify different radionuclides simultaneously present in the environment. Considering this limitation, it was decided to add new spectrometric capabilities to the GAMPIX gamma camera to achieve an imaging spectrometer. 
The Timepix chip offers two approaches for performing spectrometry measurements. The first one is based on the average size of the clusters which directly depends on the energy of the incident gamma-ray. As an example, the average cluster size is contained for a given Timepix energy threshold between 2.8 pixels for a ${ }^{241} \mathrm{Am}$ source and 7.0 pixels for a ${ }^{60} \mathrm{Co}$ source. The incident average energy can thus be deduced from the average cluster size. The second approach uses the Timepix Time over Threshold (ToT) mode [17, 22, 23]. By setting a threshold on pulses obtained at the output of charge sensitive preamplifiers, ToT mode measures the time spent by the pulses over the threshold, which is directly dependent on the incident gamma-ray energy. Conversion between cluster sizes or ToT values and energy can be achieved using reference radioactive sources or monoenergetic beams.

The purpose of this article is to demonstrate the ability of the GAMPIX system to provide spectrometric information. Qualitative and quantitative evaluation of its performances regarding this purpose will be presented. The first part of the document is dedicated to the preliminary setting of the Timepix chip and to the description of the required analysis tools. In the second part, methodology for implementing the imaging spectrometer and evaluation criteria of the final system are presented. Finally, the last part summarizes experimental results obtained in the frame of this study.

\section{Settings of the Timepix chip and analysis tools}

142

143

144

145

146

147

148

149

150

151

152

153

154

155

156

157

158

159

Fine tuning of the Timepix chip settings was crucial prior the implementation of the imaging spectrometer. It aims at optimizing both energy resolution and gain. Settings and data acquisition were performed using the Pixelman interface developed in the Czech Technical University of Prague [24]. First, threshold equalization with "noise edge" method was carried out to minimize dispersion around the average threshold value caused by gain differences between pixels. Then, a parametric study on the thirteen chip parameters showed that the $I_{\text {krum }}$ DAC had the greatest influence on both energy gain and energy resolution [25]. The $I_{\text {krum }}$ current both controls falling times of pulses generated by charge preamplifiers and compensates leakage currents (within the limit of $I_{k r u m} / 2$ ). All parameters were finally set to their default value, except $I_{k r u m}$ which was set to the DAC code value 2 corresponding to a falling time in the order of $1 \mu \mathrm{s}$ [26]. It is important to notice that the pile-up is limited with such a value. The substrate bias voltage has to be high enough (in absolute value) to minimize charge spreading and charge trapping which is a drawback of CdTe. In our case, bias voltage was set to $-110 \mathrm{~V}$. Conversion between ToT values and energy can be done by mean of a calibration curve [27-28]. This curve is mostly linear, except at very low energy (just above the threshold set on the pulses). Energy calibration also aims at optimizing energy resolution by correcting the shift between peaks due to clusters of different sizes (Fig. 2). In this study, we tested our imaging spectrometer without energy calibration but directly with ToT values. It is important to emphasize on the fact that energy resolution improvement given by the energy calibration step was not crucial for these measurements because gamma-ray spectra coming from the different studied radionuclides have a typical signature (Table I).

Data processing was performed with dedicated MATLAB software developed by CEA LIST. This software implements processing functions dealing with both spectrometric approaches 
tested in the imaging spectrometer. Concerning cluster size, the software identifies clusters as set of neighboring pixels. A maximal allowed cluster size can be set by the user to remove cosmic rays, size of which commonly exceeds 20 pixels. It is important to set a low enough acquisition time per frame to avoid pile-up which would lead to non-physical clusters resulting from the sum of successive close events. Cluster size histograms giving the number of occurrences depending on the cluster size are finally plotted. As far as ToT mode is concerned, the software sums ToT values of all pixels forming a cluster. If energy calibration has been achieved, energy conversion is done before summation. Spectra giving the number of occurrences as a function of ToT values are finally plotted. The software also achieves spatial reconstruction from the coded mask projection on the detection matrix. Spatial reconstruction can be focused on a given cluster size windowing or ToT windowing specified by the user. This functionality will be used for the implementation of the imaging spectrometer as presented in section III.

\section{Implementation of the imaging spectrometer and evaluation criteria}

179

180

181

182

183

184

185

186

187

To demonstrate the feasibility of an imaging spectrometer based of the GAMPIX gamma camera, it was decided to develop a device achieving a selective spatial reconstruction depending on the energy of incident photon (via cluster size and ToT values). This device was tested with four radioactive sources covering the energy range of interest for the GAMPIX system (see Table I). Performances of both spectrometric approaches in terms of discrimination capability, and comparison with the GAMPIX gamma camera in its current version in terms of sensitivity were assessed. The first part of this section is dedicated to the methodology used for the implementation of the imaging spectrometer while the second part justifies the choice of evaluation criteria.

\subsection{Implementation of the imaging spectrometer}

Implementation of the imaging spectrometer according to both cluster size and ToT values approaches is based on windowing. Cluster size windowing requires a preliminary measurement with each radionuclide taken alone. From the cluster size histograms, mean cluster size, dispersion around the mean and overlapping between radionuclides are evaluated. The first spatial reconstruction is performed on the single mean cluster size. Then, the windowing is progressively broadened and the best configuration is determined by qualitatively evaluated spatial reconstructions. To appreciate differences between cluster size histograms, Fig. 3 shows histograms of ${ }^{241} \mathrm{Am}$ and ${ }^{60} \mathrm{Co}$ radioactive sources and Table II gives mean cluster size and percentage of clusters in different ranges for the four radionuclides tested. Table III summarizes the cluster size windowing chosen for best discrimination. To avoid overlapping between radionuclides, mean cluster size and most frequent cluster sizes are not necessarily included in the windowing.

Concerning ToT windowing, a preliminary measurement with each radionuclide taken alone is also required to identify in the spectra ToT values associated with typical features (photoelectric peaks, Compton edge, etc.) Spectra obtained with each radionuclide are then compared to determine if there is overlapping due to the energy resolution of the sensor. The 
first windowing is centered on typical features and the best windowing is finally obtained by sequential approach. Typical features of the four radionuclides studies and position of the windowing are shown in Fig. 4. All ToT spectra obtained are in good agreement with previous literature results [27] and show the ability of the ToT mode to provide useful gamma-ray spectra, even at high energy $\left({ }^{137} \mathrm{Cs}\right.$ and $\left.{ }^{60} \mathrm{Co}\right)$. One can notice the large fraction of events on the fluorescence and escape peaks. It is explained by the pixelation of the detector: it is unusual that both incident photon and fluorescence photon deposit their energy in the same 55- $\mu \mathrm{m}$-side pixel. Table IV summarizes ToT windowing for all tested radionuclides. Because of overlapping, windowing does not necessarily include typical features.

\subsection{Evaluation criteria}

The first evaluation criterion of imaging spectrometer performances is the discrimination ability, which is qualitatively evaluated from spatial reconstructions. If radionuclides with different gamma-ray emissions are simultaneously present in the field of view, the discrimination ability characterizes the ability of the system to reconstruct only radioactive sources included in a given energy range.

The second evaluation criterion is the sensitivity, which corresponds to the minimal duration required for detecting a radionuclide inducing a given dose rate near the gamma camera. The sensitivity corresponds to a picture free of artifacts, as shown in Fig. 5 (b). Three parameters have a great impact on the sensitivity. The first one is the detector efficiency. It decreases when the incident gamma-ray energy increases as shown in Fig. 6. For a 1-mm-thick CdTe detector, efficiency drastically decreases from $100 \mathrm{keV}$. The second factor is the coded mask and its characteristics. A tradeoff has to be found between thickness (sensitivity) and number of holes (i.e. the rank, which defines the angular resolution). The last factor is the energy windowing. Without windowing (standard working mode for the GAMPIX gamma camera), all photons hitting the detector are taken into account. The narrower windowing is, the fewer photons are considered, and the more sensitivity is decreased.

\section{Performances of the imaging spectrometer}

Performances according to both evaluation criteria presented in section III are successively presented. All acquisitions were performed in "Time over Threshold" mode and in "frame" type with $1 \mathrm{~s}$ acquisition per frame. They were repeated three times to control reproducibility.

\subsection{Discrimination ability}

To evaluate discrimination capability, radioactive sources were disposed two or three at a time in front of the gamma camera over a graduated table. Tests were carried out for distance between radioactive sources and GAMPIX gamma camera varying between $50 \mathrm{~cm}$ and $150 \mathrm{~cm}$ on the camera axis and between 0 and $50 \mathrm{~cm}$ on each side of the camera on the perpendicular axis. Acquisition time was set between $300 \mathrm{~s}$ and $2000 \mathrm{~s}$ depending on configurations tested. 
Table $\mathrm{V}$ shows pictures obtained after both cluster size windowing and ToT windowing for a $2431500 \mathrm{~s}$ acquisition with ${ }^{241} \mathrm{Am},{ }^{133} \mathrm{Ba}$ and ${ }^{137} \mathrm{Cs}$ radioactive sources positioned in the configuration illustrated in Fig. 7. Both approaches are efficient for ${ }^{241} \mathrm{Am}$ and ${ }^{137} \mathrm{Cs}$ discrimination but cluster size windowing is unable to separate ${ }^{133} \mathrm{Ba}$ from ${ }^{241} \mathrm{Am} .{ }^{137} \mathrm{Cs}$ also appears less punctual for cluster size configuration and there are more artifacts on ${ }^{241} \mathrm{Am}$ picture. All tested configurations proved the superiority of ToT approach on cluster size approach regarding this evaluation criterion.

Three factors explain this superiority. First, spectra of the four radionuclides tested are well differentiated contrary to cluster size plots: centroid of photoelectric peaks varies from about 500 to 4500 from ${ }^{241} \mathrm{Am}$ to ${ }^{60} \mathrm{Co}$ (Fig. 4), while mean cluster size only changes from about 3 to 7 (Table III). Secondly, the 11810 channels of the counting system in ToT mode are great enough to show these differences. Finally, ToT mode enables to carry out fine spectrometry measurements while cluster size mode only deals with mean energy values.

\subsection{Sensitivity}

During our experiments, sensitivity was determined for each source placed at $1 \mathrm{~m}$ from the gamma camera in the camera axis, without windowing, with cluster size windowing and with ToT windowing. Several configurations of the mask were tested. We were looking for the loss of sensitivity induced by the spectro-imaging mode for both spectrometric approaches with respect to the GAMPIX gamma camera in its current version.

Tables VI to VIII summarize sensitivity for all radionuclides tested without windowing (GAMPIX gamma camera in its current version) and with cluster size and ToT windowing for several configurations of the coded mask. Percentages below the values indicate the loss of sensitivity due to both windowing techniques.

Several conclusions can be drawn from these results. Concerning coded masks, the one of rank 7 with a thickness of $4 \mathrm{~mm}$ produces best results for energies under $100 \mathrm{keV}\left({ }^{241} \mathrm{Am}\right.$, ${ }^{133} \mathrm{Ba}$ ), while mask of rank 7 with a thickness of $8 \mathrm{~mm}$ is more efficient for higher energies $\left({ }^{137} \mathrm{Cs},{ }^{60} \mathrm{Co}\right)$. Rank 13 offers better performances than rank 7 in terms of spatial resolution but is less efficient in terms of sensitivity. Two millimeters appears to be a too-thin thickness for each of the tested radionuclides, especially for high-energy gamma-ray emissions. In the case of unknown searched radionuclides, coded mask of rank 7 with a thickness of $4 \mathrm{~mm}$ offers the best tradeoff.

Concerning energy windowing, it causes a loss of sensitivity greater than $20 \%$ in most cases, which is explained by the little fraction of events occurring in the sensor and finally selected for spatial reconstruction. For low energies, this sensitivity loss is not a real problem because of very small acquisition times required by the GAMPIX gamma camera (from $1 \mathrm{~s}$ to $2 \mathrm{~s}$ for be limited by the choice of the most adapted mask. Best sensitivities are obtained for ToT windowing in comparison with cluster size windowing. Degraded results with ${ }^{60} \mathrm{Co}$ are explained by photoelectric peak spreading due to the high mean cluster size (7) and to the dispersion around this value which causes shifts between photoelectric peaks. 
The purpose of our study was to demonstrate the feasibility of an imaging spectrometer based on the GAMPIX gamma camera and to evaluate its performances. Two methods were tested to implement this imaging spectrometer: cluster size and ToT approaches. Tests on discrimination ability and sensitivity both proved the feasibility of such a device and the superiority of ToT approach. Loss of sensitivity with ToT approach is greater than $20 \%$. If it is not a problem for low energies, a relevant choice of the mask can mitigate this drawback for energies higher than $100 \mathrm{keV}$. If the radionuclide is unknown, the coded mask of rank 7 with a thickness of $4 \mathrm{~mm}$ offers the best compromise.

Further developments of the imaging spectrometer will combine both cluster size and ToT spectrometric approaches. Next planned step is the integration of the energy calibration in the imaging spectrometer for the analysis of closer gamma-ray energies. Because of the high mean cluster size, improvements are also expected for high energies $\left({ }^{60} \mathrm{Co}\right)$. Energy calibration measurements should take place at the SOLEX facility which provides monoenergetic beams from $0.5 \mathrm{keV}$ to $28 \mathrm{keV}$ [30]. As a first step, we plan the global calibration of the Timepix chip. Improvements of the energy resolution of a factor between two and four are reported by [26] with a pixel by pixel calibration and this approach will be considered as a second step. Test of a $1 \mathrm{~mm}$ thick, $110 \mu \mathrm{m}$ pixel side Timepix chip is also expected. It would enable to evaluate the energy resolution gain due to the limitation of charge sharing between several pixels, which is one of the main explanations for energy resolution degradation.

Finally, the replacement of the Timepix chip by a Medipix 3 chip will be studied. The ToT mode is not implemented in the Medipix 3 chip and spectra have to be obtained by counting the number of events for each threshold value [31]. The main improvement compared to previous Medipix chips concerns the hardware connection between several neighboring pixels, which should drastically improve the energy resolution of the system.

\section{References}

[1] F. Carrel, et al., "GAMPIX: a New Gamma Imaging System for Radiological Safety and Homeland Security Purposes", IEEE NSS Conference Record, 2011.

[2] V. Schoepff, et al., "BOOSTER: Development of a Toolbox for Triage of Large Group of Individuals Exposed to Radioactive Material", accepted for publication in IEEE Transactions on Nuclear Science, 2014.

[3] W. Kaye, et al., "Calibration and Operation of the Polaris 18-Detector CdZnTe Array", IEEE, 2010.

[4], "Polaris-H Gamma-Ray Imaging Spectrometer", presentation brochure.

[5] H.O. Anger, "Scintillation Camera", The review of scientific instruments, vol. 29, num. 1, 1958.

[6] M. Ricard, "Imaging of gamma emitters using scintillation cameras", Nucl. Instrum. Methods Phys. Res. A, vol. 527, pp. 124-129, 2004. 
[7] O. Gal, et al., "CARTOGAM - a portable gamma camera for remote localization of radioactive sources in nuclear facilities", Nucl. Instrum. Methods Phys. Res. A, vol. 460, pp. 138-145, 2001.

[8] S.V. Guru, et al., "A high energy gamma camera using a multiple hole collimator and PSPMT”, Nucl. Instrum. Methods Phys. Res. A, vol. 353, pp. 328-333, 1994.

[9] M. Woodring, et al., "Advanced radiation imaging of low-intensity gamma-ray sources", Nucl. Instrum. Methods Phys. Res. A, vol. 422, pp. 709-712, 1999.

[10] Z. He, et al., “The CSPD-2 Gamma-Ray Imaging System”, IEEE TNS, vol. 44, num. 3, 1997.

[11] R. Redus, et al., "A combined video and gamma ray imaging system for robots in nuclear environments", Nucl. Instrum. Methods Phys. Res. A, vol. 353, pp. 324-327, 1994.

[12] V.R. McCready, et al., "Clinical tests on a prototype semiconductor gamma-camera", British Journal of Radiology, vol. 44, pp. 58-62, 1971.

[13] Y. Eisen, et al.., "A gamma camera based on CdTe detector", Nucl. Instrum. Methods Phys. Res. A, vol. 380, pp. 474-478, 1996.

[14] Z. He, et al., "3-D position sensitive CdZnTe gamma-ray spectrometers", Nucl. Instrum. Methods Phys. Res. A, vol. 422, pp. 173-178, 1999.

[15] C. Mestais, et al., "A new design for a high resolution, high efficiency CZT gamma camera detector", Nucl. Instrum. Methods Phys. Res. A, vol. 458, pp. 62-67, 2001.

[16] O. Limousin, et al., "Caliste 256: A CdTe imaging spectrometer for space science with a $580 \mu \mathrm{m}$ pixel pitch", Nucl. Instrum. Methods Phys. Res. A, vol. 647, pp. 46-54, 2011.

[17] X. Llopart, et al., "Timepix, a 65k programmable pixel readout chip for arrival time, energy and/or photon counting", Nucl. Instrum. Methods Phys. Res. A, vol. 581, pp. 485494, 2007.

[18] M. Gmar, et al., "GAMPIX: a new generation of gamma camera", Nucl. Instrum. Methods Phys. Res. A, Vol. 652, pp. 638-640, 2011.

[19] F. Carrel, et al., "GAMPIX: a new generation of gamma camera for hot spot localisation", Proceedings of the ISOE Conference, Cambridge, 2010.

[20] S. R. Gottesman and E. E. Fenimore, "New family of Binary Arrays for Coded Aperture Imaging”, Applied Optics, Vol. 28, n. 20, pp. 4344-4352, 1989.

[21] Z. Vykydal, "Microprocessor controlled USB interface for Medipix2 detector", Ph.D. dissertation, Department of Physical Electronics, Czech Technical University, Prague, Poland, 2004-2005.

[22] D. Maneuski, et al., "Imaging and spectroscopic performance studies of pixelated CdTe detector", Journal of Instrumentation, vol. 7, 2012.

[23] E. Fröjdh, et al., "X-ray absorption and charge transport in a pixelated CdTe detector with single photon processing readout", Journal of Instrumentation, Vol. 7, 2011.

[24] D. Turecek, et al., "Pixelman: a multi-platform data acquisition and processing software package for Medipix2, Timepix and Medipix3 detectors", IOP, 2011.

[25] M. Kroupa, et al., "Optimization of the spectroscopic response of the Timepix detector", IOP, 2011.

[26] M. Filipenko et al., "Characterization of the energy resolution and the tracking capabilities of a hybrid pixel detector with CdTe-sensor layer for a possible use in a neutrinoless double beta decay experiment”, Eur. Phys. J. C, 73:2374, 2013. 
367 [27] J. Jakubek, "Precise energy calibration of pixel detector working in time-over-threshold mode", Nucl. Instrum. Methods Phys. Res. A, vol. 633, pp. S262-S266, 2011.

369 [28] D. Turecek, et al., "Energy calibration of pixel detector working on Time-OverThreshold mode using test pulses”, NSS/MIC ISSN 1082-3654, pp. 1722-1725, 2011.

[29] C. Ponchut and M. Ruat, "Energy calibration of a CdTe X-ray pixel sensor hybridized to a Timepix chip", IOP, 2012.

[30] Y. Ménesguen and M.C. Lépy, "Efficiency calibration and surface mapping of an energy-dispersive detector with SOLEX: a compact tunable monochromatic X-ray source”, Nucl. Instrum. Methods Phys. Res. A, vol. 695, pp. 193-196, 2012.

[31] R. Ballabriga et al., "Medipix3:A $64 \mathrm{k}$ pixel detector readout chip working in single photon counting mode with improved spectrometric performance", Nucl. Instrum. Methods Phys. Res. A, vol. 633, pp. S15-S18, 2011. 


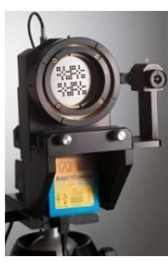

(a)

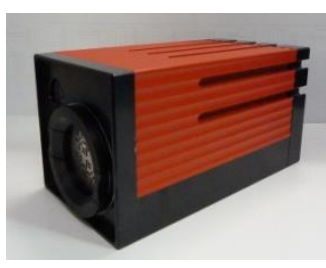

(b)

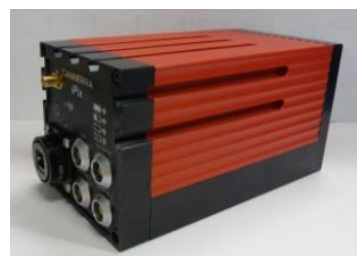

(c)

381 Fig. 1: (a) GAMPIX gamma camera prototype developed by CEA LIST (b) front side and (c) back side of the 382 iPix industrial prototype developed by CANBERRA.

383

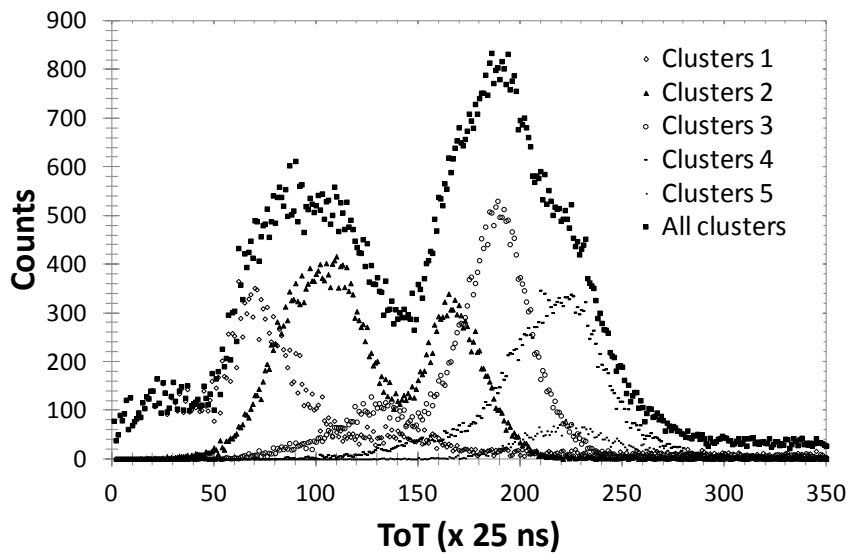

385 Fig. 2: Spectra obtained with a ${ }^{241}$ Am radioactive source depending on cluster size.

386

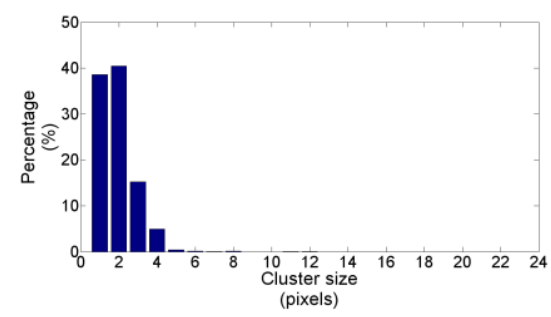

(a)

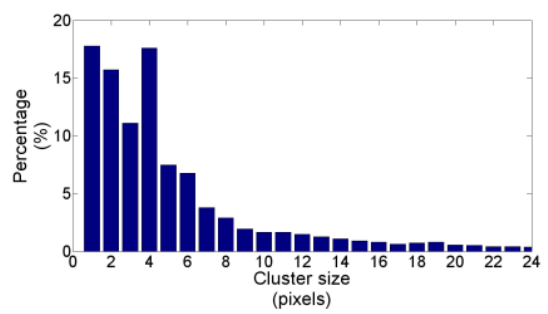

(b)

387 Fig. 3: Cluster size histograms for (a) ${ }^{241} \mathrm{Am}$ and (b) ${ }^{60} \mathrm{Co}$

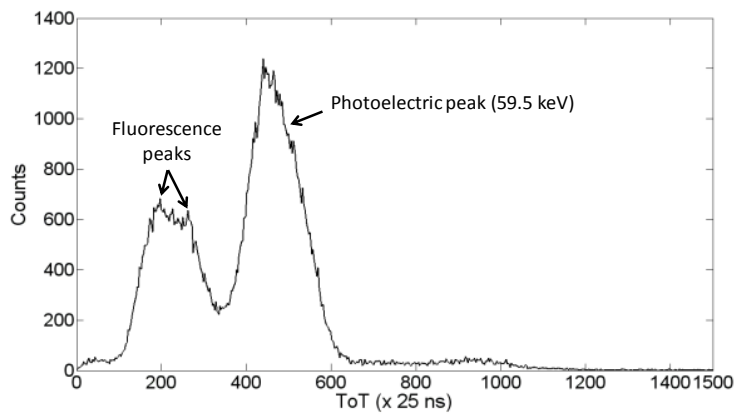



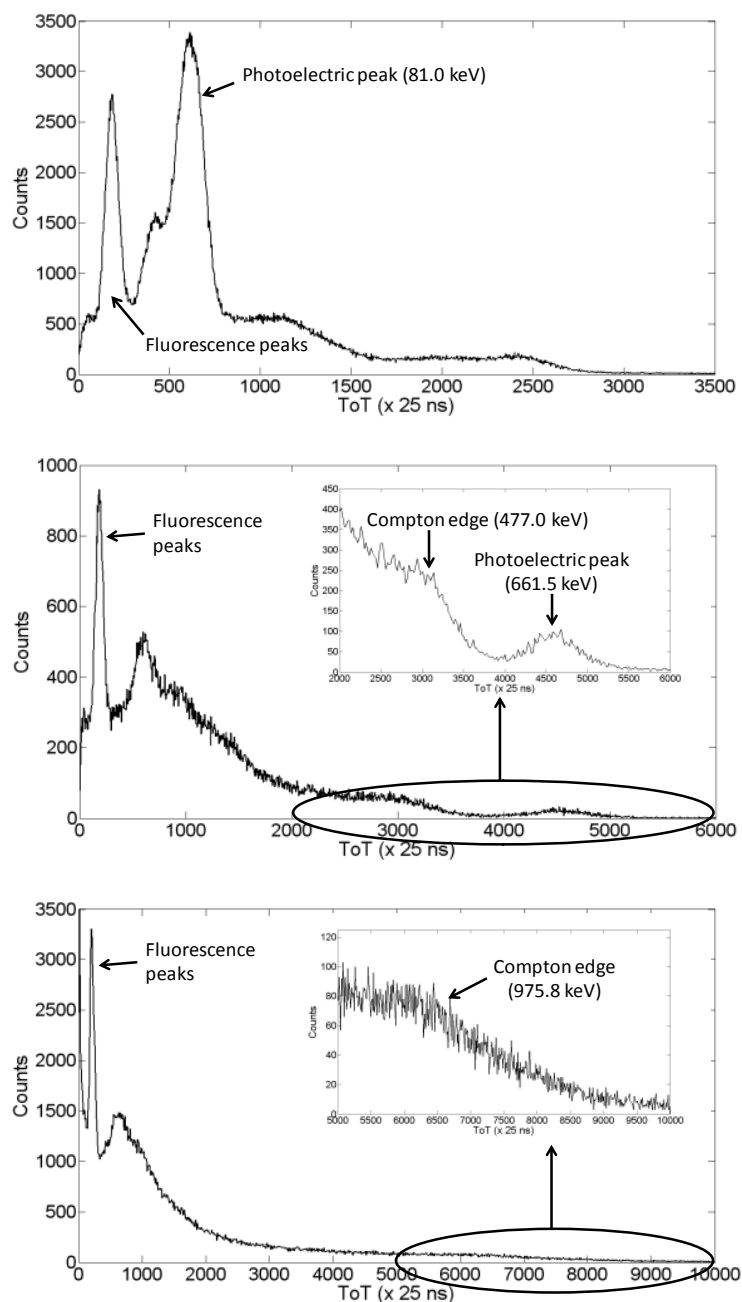

(d)

Fig. 4: Spectra of (a) ${ }^{241} \mathrm{Am}$, (b) ${ }^{133} \mathrm{Ba}$, (c) ${ }^{137} \mathrm{Cs}$ and (d) ${ }^{60} \mathrm{Co}$ with typical features.

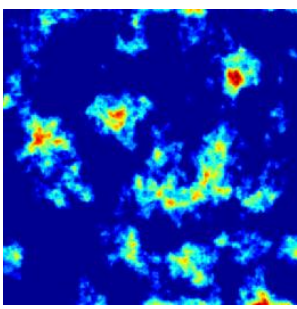

(a)

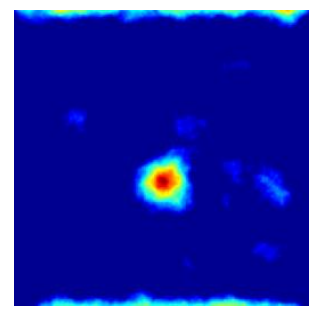

(b)

391 Fig. 5: Spatial reconstruction of a radioactive source of ${ }^{137} \mathrm{Cs}$ (coded mask of rank 7 with thickness of $4 \mathrm{~mm}$ ) for acquisition time of (a) $10 \mathrm{~s}$ and (b) $400 \mathrm{~s}$. The presence of artifacts can be observed on the left. Result obtained for a $400 \mathrm{~s}$ acquisition time is considered as satisfying. 


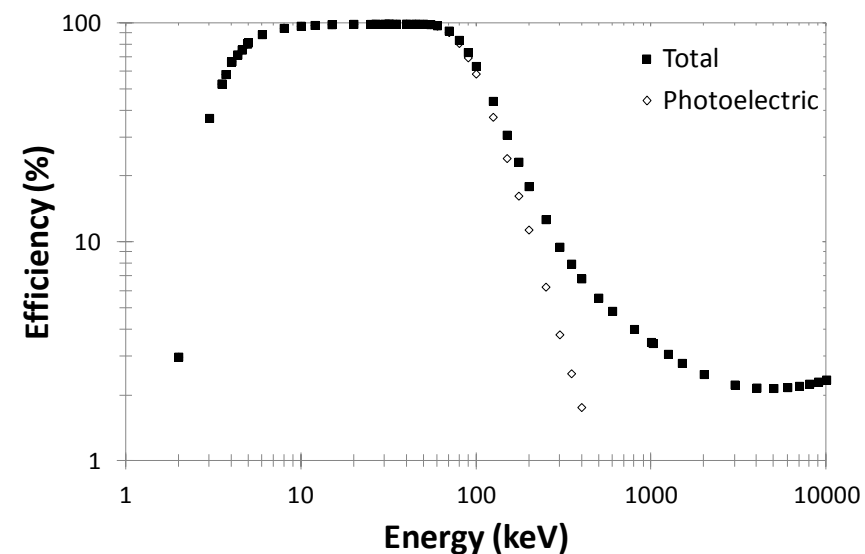

395
Fig. 6: Logarithmic plot of efficiency for $1 \mathrm{~mm}$ CdTe detector between $1 \mathrm{keV}$ and $10 \mathrm{MeV}$.

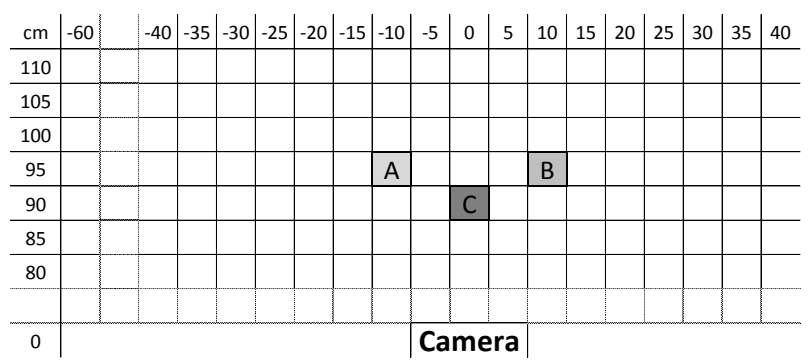

\section{$\mathrm{A}^{241} \mathrm{Am}$ $\mathrm{B}{ }^{133} \mathrm{Ba}$ C ${ }^{137} \mathrm{Cs}$}

Table 1

Characteristics of radioactive sources tested

\begin{tabular}{lcccc} 
Source & ${ }^{241} \mathrm{Am}$ & ${ }^{133} \mathrm{Ba}$ & ${ }^{137} \mathrm{Cs}$ & ${ }^{60} \mathrm{Co}$ \\
\hline Energy $(\mathrm{keV})$ & 59.5 & {$[81.0,356.0]$} & 661.7 & 1173.2 and 1332.5 \\
Activity $(\mathrm{MBq})$ & 72.8 & 35.1 & 26.1 & 11.7 \\
Dose rate at $1 \mathrm{~m}(\mu \mathrm{Sv} / \mathrm{h})$ & 0.286 & 1.658 & 1.987 & 3.585
\end{tabular}

Table 2

Mean cluster size and cluster size probabilities

\begin{tabular}{ccccc}
\multirow{2}{*}{ Radionuclide } & Mean cluster size (pixels) & \multicolumn{3}{c}{ Cluster size probability (\%) } \\
& & $0-4$ & $4-10$ & $>10$ \\
\hline${ }^{241} \mathrm{Am}$ & 2.8 & 90.1 & 9.8 & 0.1 \\
${ }^{133} \mathrm{Ba}$ & 3.9 & 70.4 & 28.4 & 1.2 \\
${ }^{137} \mathrm{Cs}$ & 4.4 & 59.9 & 35.4 & 4.7 \\
${ }^{60} \mathrm{Co}$ & 7.0 & 54.6 & 30.8 & 14.6
\end{tabular}

Table 3

Cluster size windowing

\begin{tabular}{ccc} 
Radionuclide & Windowing (cluster size) & Mean cluster size (pixels) \\
\hline${ }^{241} \mathrm{Am}$ & $0-3$ & 2.8 \\
${ }^{133} \mathrm{Ba}$ & $7-10$ & 3.9 \\
${ }^{137} \mathrm{Cs}$ & $10-100$ & 4.4 \\
${ }^{60} \mathrm{Co}$ & $12-100$ & 7.0
\end{tabular}


413

414

415

416

417

418

419

420

421

422

423

424

425
Table 4

ToT windowing

\begin{tabular}{cc} 
Radionuclide & Windowing (ToT values) \\
\hline${ }^{241} \mathrm{Am}$ & $400-500$ \\
${ }^{133} \mathrm{Ba}$ & $600-800$ \\
${ }^{137} \mathrm{Cs}$ & $1000-2000$ \\
${ }^{60} \mathrm{Co}$ & $5000-15000$
\end{tabular}

Table 5

Mean cluster size and cluster windowing

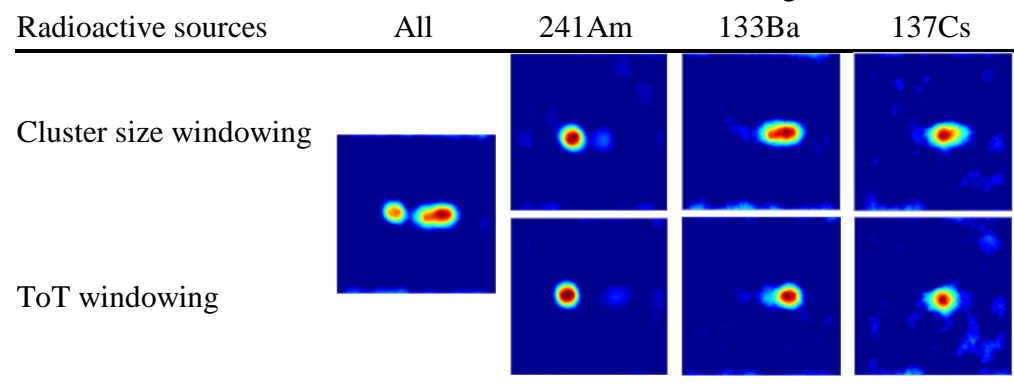

Table 6

Sensitivities without windowing and for cluster size and ToT windowing with rank 7, thickness of $4 \mathrm{~mm}$ coded mask

\begin{tabular}{cccc} 
& Without windowing & Cluster size windowing & ToT windowing \\
\hline${ }^{241} \mathrm{Am}$ & $1 \mathrm{~s}$ & $1 \mathrm{~s}$ & $\mathbf{1 ~ s}$ \\
& & $0 \%$ & $\mathbf{0 \%}$ \\
${ }^{133} \mathrm{Ba}$ & $4 \mathrm{~s}$ & $15 \mathrm{~s}$ & $\mathbf{7 ~ s}$ \\
& & $+275 \%$ & $+\mathbf{7 5 \%}$ \\
${ }^{137} \mathrm{Cs}$ & $60 \mathrm{~s}$ & $130 \mathrm{~s}$ & $100 \mathrm{~s}$ \\
& & $+117 \%$ & $+67 \%$ \\
${ }^{60} \mathrm{Co}$ & $300 \mathrm{~s}$ & $400 \mathrm{~s}$ & $1500 \mathrm{~s}$ \\
& & $+33 \%$ & $+400 \%$
\end{tabular}

Table 7

Sensitivities without windowing and for cluster size and ToT windowing with rank 7, thickness of $8 \mathrm{~mm}$ coded mask

\begin{tabular}{cccc} 
& Without windowing & Cluster size windowing & ToT windowing \\
\hline${ }^{241} \mathrm{Am}$ & $1 \mathrm{~s}$ & $2 \mathrm{~s}$ & $2 \mathrm{~s}$ \\
& & $+100 \%$ & $+100 \%$ \\
${ }^{133} \mathrm{Ba}$ & $10 \mathrm{~s}$ & $25 \mathrm{~s}$ & $12 \mathrm{~s}$ \\
& & $+150 \%$ & $+20 \%$ \\
${ }^{137} \mathrm{Cs}$ & $20 \mathrm{~s}$ & $180 \mathrm{~s}$ & $\mathbf{8 0 ~ s}$ \\
& & $+800 \%$ & $+\mathbf{3 0 0 \%}$ \\
${ }^{60} \mathrm{Co}$ & $60 \mathrm{~s}$ & $\mathbf{1 2 5} \mathrm{s}$ & $650 \mathrm{~s}$ \\
& & $\mathbf{+ 1 0 8 \%}$ & $+983 \%$
\end{tabular}

426

427

428 
Table 8

430 Sensitivities without windowing and for cluster size and ToT windowing with rank 13, thickness of 2 mm coded 431 mask

Without windowing Cluster size windowing ToT windowing

432

\begin{tabular}{cccc} 
& Without windowing & Cluster size windowing & ToT windowing \\
\hline${ }^{241} \mathrm{Am}$ & $3 \mathrm{~s}$ & $4 \mathrm{~s}$ & $4 \mathrm{~s}$ \\
& & $+33 \%$ & $+33 \%$ \\
& & $100 \mathrm{~s}$ & $17 \mathrm{~s}$ \\
${ }^{133} \mathrm{Ba}$ & $14 \mathrm{~s}$ & $+614 \%$ & $+21 \%$ \\
& & $>600 \mathrm{~s}$ & $>600 \mathrm{~s}$ \\
${ }^{137} \mathrm{Cs}$ & $300 \mathrm{~s}$ & $>+100 \%$ & $>+100 \%$ \\
& & Not visible & Not visible
\end{tabular}

